\title{
Intra-species variation within Lactobacillus rhamnosus correlates to beneficial or harmful outcomes: lessons from the oral cavity
}

\author{
Mangala A. Nadkarni ${ }^{1,2,3^{*}}$, Nandan P. Deshpande ${ }^{4+}$, Marc R. Wilkins ${ }^{4,5,6}$ and Neil Hunter ${ }^{1,2}$
}

\begin{abstract}
Background: The origin of most of the Lactobacillus rhamnosus genome sequences lodged in NCBI can be traced to food and faecal isolates followed by blood and tissue sites but with minimal representation from oral and vaginal isolates. However, on the L. rhamnosus phylogenetic tree no apparent clade is linked to the origin of isolation or to the relevant clinical source, except for a distinct clade exclusively shared by L. rhamnosus isolates from early stages of dental pulp infection (LRHMDP2 and LRHMDP3) and from bronchoalveolar lavage (699_LRHA and 708_LRHA) from a critical care patient. These L. rhamnosus strains, LRHMDP2, LRHMDP3, 699_LRHA and 708_ LRHA isolated from different continents, display closest genome neighbour gapped identity of $99.95 \%$. The aim of this study was to define a potentially unique complement of genes of clinical relevance shared between these $L$. rhamnosus clinical isolates in comparison to probiotic L. rhamnosus strains.

Results: In this analysis we used orthologous protein identification tools such as ProteinOrtho followed by tblastn alignments to identify a novel tyrosine protein phosphatase (wzb)-tyrosine-protein kinase modulator EpsC (wzd)synteny exopolysaccharide (EPS) cluster. This EPS cluster was specifically conserved in a clade of 5 clinical isolates containing the four L. rhamnosus clinical isolates noted above and Lactobacillus spp. HMSC077C11, a clinical isolate from a neck abscess. The EPS cluster was shared with only two other strains, L. rhamnosus BPL5 and BPL15, which formed a distant clade on the L. rhamnosus phylogenetic tree, with a closest genome neighbour gapped identity of 97.51\% with L. rhamnosus LRHMDP2 and LRHMDP3.

Exclusivity of this EPS cluster (from those identified before) was defined by five EPS genes, which were specifically conserved between the clade of 5 clinical isolates and L. rhamnosus BPL5 and BPL15 when compared to the remaining $L$. rhamnosus strains. Comparative genome analysis between the clade of 5 clinical isolates and $L$. rhamnosus BPL5 and BPL15 showed a set of 58 potentially unique genes characteristic of the clade of 5.

Conclusion: The potentially unique functional protein orthologs associated with the clade of 5 clinical isolates may provide understanding of fitness under selective pressure.
\end{abstract}

Keywords: Lactobacillus rhamnosus, Dental caries, Infection, Genome, Defense, Toxin-antitoxin, Extracellular polysaccharide

\footnotetext{
* Correspondence: mangala.nadkarni@health.nsw.gov.au

Mangala A. Nadkarni and Nandan P. Deshpande joint first

${ }^{1}$ Institute of Dental Research, Westmead Centre for Oral Health, Westmead

Hospital, Sydney, New South Wales, Australia

${ }^{2}$ Westmead Institute for Medical Research, The University of Sydney, Sydney,

New South Wales, Australia

Full list of author information is available at the end of the article
}

(C) The Author(s). 2020 Open Access This article is licensed under a Creative Commons Attribution 4.0 International License, which permits use, sharing, adaptation, distribution and reproduction in any medium or format, as long as you give appropriate credit to the original author(s) and the source, provide a link to the Creative Commons licence, and indicate if changes were made. The images or other third party material in this article are included in the article's Creative Commons licence, unless indicated otherwise in a credit line to the material. If material is not included in the article's Creative Commons licence and your intended use is not permitted by statutory regulation or exceeds the permitted use, you will need to obtain permission directly from the copyright holder. To view a copy of this licence, visit http://creativecommons.org/licenses/by/4.0/. The Creative Commons Public Domain Dedication waiver (http://creativecommons.org/publicdomain/zero/1.0/) applies to the data made available in this article, unless otherwise stated in a credit line to the data. 


\section{Background}

The ubiquitous nature of $L$. rhamnosus in multiple ecological niches including food, gut, oral cavity, vaginal cavity and other tissue sites has inspired many studies of the evolution, niche adaptability and possible safety concerns relating to occasional opportunistic pathogenicity of this species [1-4].

Blood isolates of L. rhamnosus [5] and clinical isolates from intensive care unit (ICU) patients [6] showed no apparent clustering on the L. rhamnosus phylogenetic tree, while a grouping was reported between L. rhamnosus strains from food and faeces [3] within the limited numbers of L. rhamnosus genomes accessible through NCBI at the time of that report (2014). Between 2009 and 2019, $172 \mathrm{~L}$. rhamnosus genome assemblies became available in NCBI providing an opportunity to review clustering within this species.

Analysis indicated that L. rhamnosus HN001 from a yoghurt inoculum was seen to group with L. rhamnosus E800 isolated from human faeces. L. rhamnosus R011 from cheddar cheese grouped with L. rhamnosus ATCC 21052 from faeces while L. rhamnosus LC705 from milk grouped with L. rhamnosus ATCC 8530 isolated from human airways and L. rhamnosus LMS2-1 from human gut [3]. Probiotic lactobacilli, L. rhamnosus GG and $L$. rhamnosus 53103, isolated from the gut of a healthy individual, grouped with PEL5 and PEL6 sourced from a gut biopsy [3]. It was notable that L. rhamnosus LRHM DP2 and L. rhamnosus LRHMDP3, isolated from the early stages of infection of dental pulp, formed a distinct cluster $[3,7]$. These strains could be categorized as having invasive potential as a significant role for L. rhamnosus in the early stages of invasion of vital pulp tissue became apparent in 16S rRNA-based fluorescence insitu hybridization studies [8]. In the oral cavity, L. rhamnosus was implicated in the progression of cavitated carious lesions. Accordingly, the organism was distributed over a broad $\mathrm{pH}$ range from an acidic superficial zone of decalcified dentine matrix to a higher $\mathrm{pH}$ zone adjacent to vital dental pulp [9]. However, in the initial stages of dental pulp infection the abundance of $L$. rhamnosus expressing copious amounts of exopolysaccharide suggested a pathogenic potential beyond the capacity for production of lactic acid [8, 10-13]. L. rhamnosus LRHMDP2 and L. rhamnosus LRHMDP3, were the first of the oral isolates to be sequenced $[3,7]$. These isolates were segregated from the probiotic strain L. rhamnosus GG by 264 and 258 new genes respectively. Differences included a distinctive exopolysaccharide cluster (EPS), transcriptional regulators, an iron $\mathrm{ABC}$ transporter and a two component sensor kinase with Ferric iron transporter. Additional differences included the absence of $L$. rhamnosus GG spaCBA pilus cluster and of the Clustered Regularly Interspaced Short Palindromic Repeat
(CRISPR) - CRISPR associated (cas) system [7]. Genome sequences from $18 \mathrm{~L}$. rhamnosus clinical isolates from ICU patients [6] revealed that two L. rhamnosus clinical isolates, 699_LRHA and 708_LRHA, isolated 4 days apart from bronchoalveolar lavage of the same patient, were the only clinical isolates to exclusively share the distinct clade with LRHMDP2 and LRHMDP3. Other oral isolates, L. rhamnosus LRB from an exfoliated deciduous tooth [14] and L. rhamnosus 24, 308 and 313 from infant and adult saliva (closest genome neighbour gapped identity of $97.4 \%$ with $L$. rhamnosus LRHMDP2 and LRHMDP3) as well as other clinical isolates from a variety of tissue sources, showed random distribution on the L. rhamnosus phylogenetic tree as displayed in Fig. 1.

Apart from the significant association of L. rhamnosus with progression of dental caries, and as an occasional opportunistic pathogen in infective endocarditis in patients with cardiac risk factors $[15,16]$, a major focus on L. rhamnosus has been on probiotic properties. One of the criteria to be generally recognized as safe (GRAS) and having beneficial effects on the host, is the absence of an inflammatory response [17]. Accordingly, the focus on probiotic action of lactobacilli in humans is on released products and on surface components, particularly exopolysaccharides (EPS) of capsular or cell wall origin [18]. Probiotic L. rhamnosus LOCK 900 has been shown to express both low and high molecular weight exopolysaccharides with distinct structures and biological properties [19]. Similarly, the genome of L. rhamnosus DSM 14870 from the prophylactic EcoVag capsule was found to encode two putative EPS clusters. EPS cluster 1 exhibited identity to L. rhamnosus Lc705 EPS cluster while the majority of the ORFs of the EPS cluster 2 showed identity to L. rhamnosus HN001 EPS cluster [20]. Similarly, EPS clusters of other L. rhamnosus probiotic and clinical isolates have also shown considerable divergence $[7,18,21,22]$. Blood isolates from cluster A $(n=8)$ shared the EPS cluster of $L$. rhamnosus GG whereas other blood isolates from cluster B $(n=7)$ were found to possess a different EPS cluster [5]. L. rhamnosus strains $(n=40)$ from diverse environments such as fermented dairy products, beer, animal and human faeces, blood and vagina, group into six EPS gene clusters with one of the EPS clusters formed by multiple predicted mannosyl-glycosyltransferases considered to synthesise mannosyl-EPS [1]. Beyond the focus on the EPS cluster, niche adaptation was also attributed to an accessory genome comprising pilus gene clusters, CRISPR - cas system genes, carbohydrate transport and metabolism genes, bacteriocin production and mobile genetic elements [1].

In the present study, using in-silico analysis, we highlight a potentially unique complement of genes of 


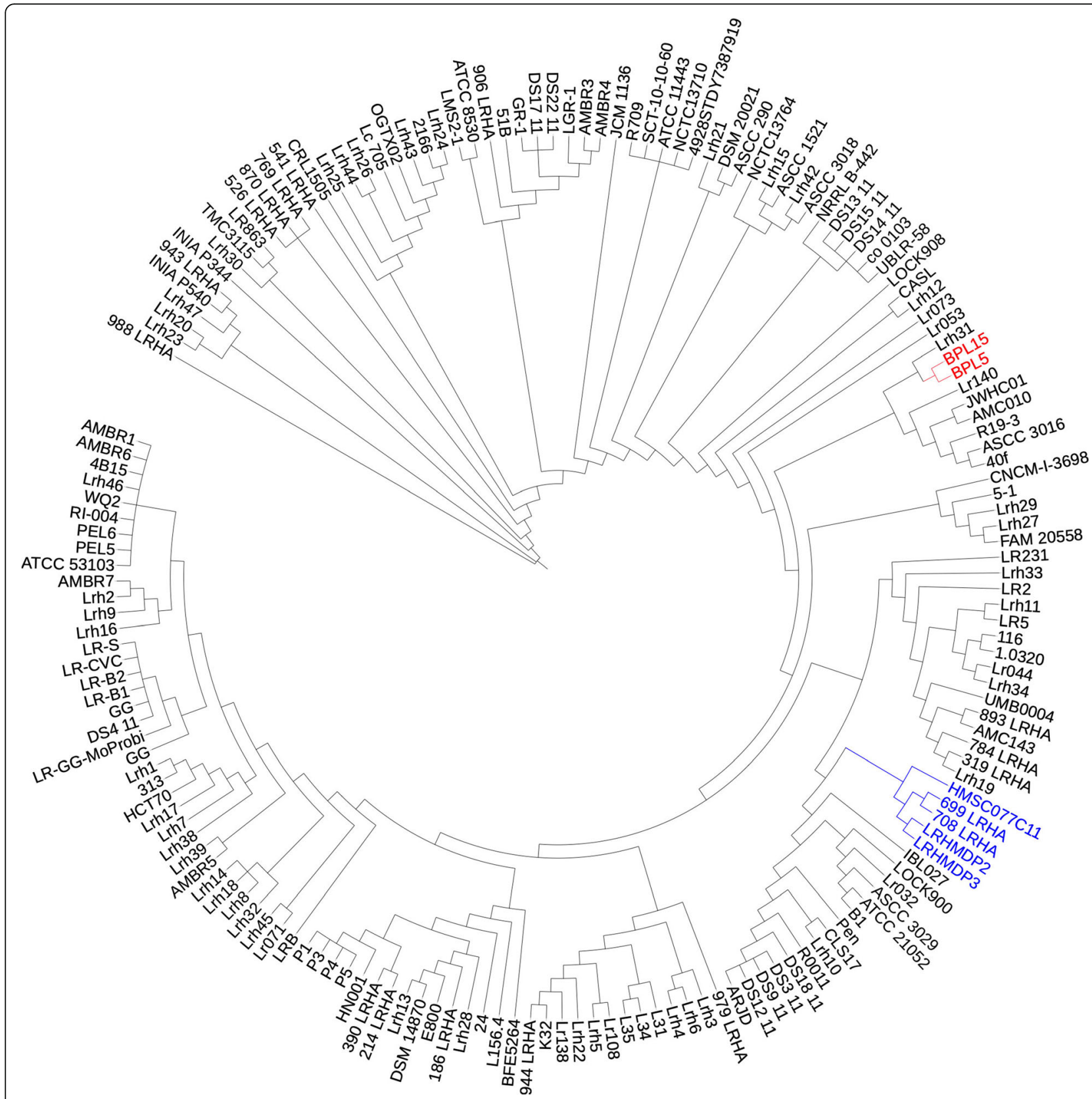

Fig. 1 L. rhamnosus phylogenetic tree. GToTree was used to generate alignments and a phylogenetic tree based on HMM profiles. For comparative genomics analysis Genbank, fasta and gff format files were downloaded using the NCBI web link https://www.ncbi.nlm.nih.gov/ genome/doc/ftpfaq/\#downloadservice (Table S1). Blue: Genome sequences of L. rhamnosus and Lactobacillus spp. from the exclusive clinical clade of 5 strains: L. rhamnosus LRHMDP2, LRHMDP3, 699_LRHA, 708_LRHA and Lactobacillus spp. HMSC077C11. Red: L. rhamnosus BPL5 and BPL15 form a clade distant from the clinical clade of 5 strains

clinical relevance in the clade of 5 clinical isolates $(L$. rhamnosus LRHMDP2, LRHMDP3, 699_LRHA, 708_ LRHA and Lactobacillus spp. HMSC077C11) in comparison to the probiotic strains L. rhamnosus BPL5 and BPL15. Except for the near identical EPS cluster, with exclusivity defined by five EPS orthologs, no other genes were specifically conserved between the clade of 5 clinical isolates and the probiotic strains, L. rhamnosus
BPL5 and BPL15 as compared to other L. rhamnosus strains.

A set of 58 genes with known biological functions were found to be specifically conserved across the clade of 5 clinical isolates when compared to the L. rhamnosus BPL5 and BPL15 strains. Of the 58 genes, 14 genes with important biological functions were found to be orthologous across the clade of 5 clinical isolates with minimal 
presence (1-7 strains) across the remaining L. rhamnosus strains.

\section{Results}

A unique clade of 5 clinical isolates of $L$. rhamnosus

With no apparent clades reflecting the origin of isolation within a phylogeny tree comprising 172 sequenced isolates (Fig. 1, Table S1), it was considered important to decipher the genomic features of $L$. rhamnosus isolates that represented a unique clade on the L. rhamnosus phylogenetic tree.

L. rhamnosus isolates from the early stages of dental pulp infection (LRHMDP2 and LRHMDP3) which showed closest genome neighbour gapped identity of 99.95\% with L. rhamnosus isolates (699_LRHA and 708_ LRHA) from bronchoalveolar lavage, shared a distinct clade on the L. rhamnosus phylogeny tree (Fig. 1). L. rhamnosus 699_LRHA and 708_LRHA were isolated 4 days apart from the same patient from a polymicrobial infection that included Escherichia coli and Serratia marcescens [6]. Inclusion of Lactobacillus spp. HMSC077C11 in the present study was incidental based on an identical protein search on NCBI for the L. rhamnosus LRHMDP2 and LRHMDP3 EPS cluster protein orthologs (Table 1). The Lactobacillus spp. HMSC077C11 genome sequence was lodged in NCBI as an unnamed isolate not characterized using traditional culture identification methods and being clearly distinct from currently identified species (https://www.ncbi.nlm. nih.gov/genome/?term=HMSC077C11). However, a recent genome- based species taxonomy study re-classified Lactobacillus spp. HMSC077C11 as Lactobacillus rhamnosus in the Genome Taxonomy Database (GTDB) [23]. Our analysis also showed that Lactobacillus spp. HMSC077C11 occupied the same clade as L. rhamnosus LRHMDP2, LRHMDP3, 699_LRHA and 708_LRHA on the L. rhamnosus phylogenetic tree (Fig. 1).

\section{A novel EPS cluster shared across the clade of $5 L$. rhamnosus clinical isolates and the $L$. rhamnosus strains BPL5 and BPL15}

A targeted search on NCBI for Identical Protein groups of L. rhamnosus LRHMDP2 and LRHMDP3 wzb-wzd synteny EPS cluster led to the finding of a conserved EPS cluster in the clade of 5 clinical isolates. This finding offered a critical basis to search for conservation of the EPS cluster orthologs across other L. rhamnosus strains. Of the $172 \mathrm{~L}$. rhamnosus isolates whose sequences are included in this study, only two others, $L$. rhamnosus strains, BPL5 (CECT 8800) a vaginal probiotic [24] and L. rhamnosus BPL15 (CECT 8361) [25], which form a distant clade, shared a near identical EPS cluster with the clade of 5 clinical isolates. Both $L$. rhamnosus strains, BPL5 and BPL15, showed a closest genome neighbour gapped identity of $97.51 \%$ with $L$. rhamnosus LRHMDP2 and LRHMDP3 and 97.49\% with L. rhamnosus 699_LRHA and 708_LRHA. Therefore, these two strains were also included in the analysis.

Five genes from the wzb-wzd bound EPS cluster, a gene encoding a homologue of lipopolysaccharide biosynthesis protein, a glycosyltransferase family 2 protein (wchA), a polysaccharide pyruvyl transferase family protein, a hypothetical protein and an oligosaccharide repeat unit polymerase, were syntenically conserved specifically in these seven strains (the clade of 5 clinical isolates and L. rhamnosus BPL5 and BPL15) when compared to all other L. rhamnosus strains (Table 1). The five genes are thus exclusive to this EPS cluster. The remainder of the elements of the wzb-wzd EPS cluster were found to be conserved across many other L. rhamnosus strains. The tool EasyFig was used to ascertain the genomic organization of EPS cluster genes displaying similarities and differences in the orientation within the 'wzb-wzd' synteny EPS cluster across the clade of 5 clinical isolates and BPL5 and BPL15 (Fig. 2a, b, c. The green and orange colours specify gene orientations). Near identical genomic organisation and orientation of EPS cluster genes between the two dental pulp isolates, L. rhamnosus LRHMDP2 and LRHMDP3, was apparent [7] except for an inversion in the intergenic region adjacent to the welE gene and the presence of an additional hypothetical protein in LRHMDP2 as compared to LRHMDP3 (Fig. 2a). Similarly, genomic organisation and orientation of EPS cluster genes between L. rhamnosus BPL5 and BPL15 also remained near identical but for an inversion in the intergenic region (adjacent to the welE gene) as was apparent in LRHMDP2 and LRHM DP3 (Fig. 2a). Lactobacillus spp. HMSC077C11 EPS cluster genes showed consensus with L. rhamnosus BPL5 and BPL15 for genomic organisation and orientation except for a missing gene adjacent to the inversion region (Fig. 2a). However, the EPS cluster genes of $L$. rhamnosus BPL5, BPL15 and Lactobacillus spp. HMSC077C11 showed inverted orientation as compared to L. rhamnosus LRHMDP2 and LRHMDP3 (Fig. 2a). When the genomic context of the relevant assemblies was examined, the EPS cluster genes of L. rhamnosus LRHMDP2, LRHMDP3 and Lactobacillus spp. HMSC077C11 could be detected on the same contig whereas EPS cluster genes of L. rhamnosus 699_LRHA were found to be split between two different contigs (Fig. 2b) and 708_LRHA EPS cluster genes were found split between three different contigs (Fig. 2c). In L. rhamnosus 699_LRHA orientation of some of the EPS cluster genes was inverted compared to L. rhamnosus LRHMDP2 and LRHMDP3. L. rhamnosus 708_LRHA maintained orientation of the EPS cluster genes in the same order as for $L$. rhamnosus LRHMDP2 and LRHMDP3. 


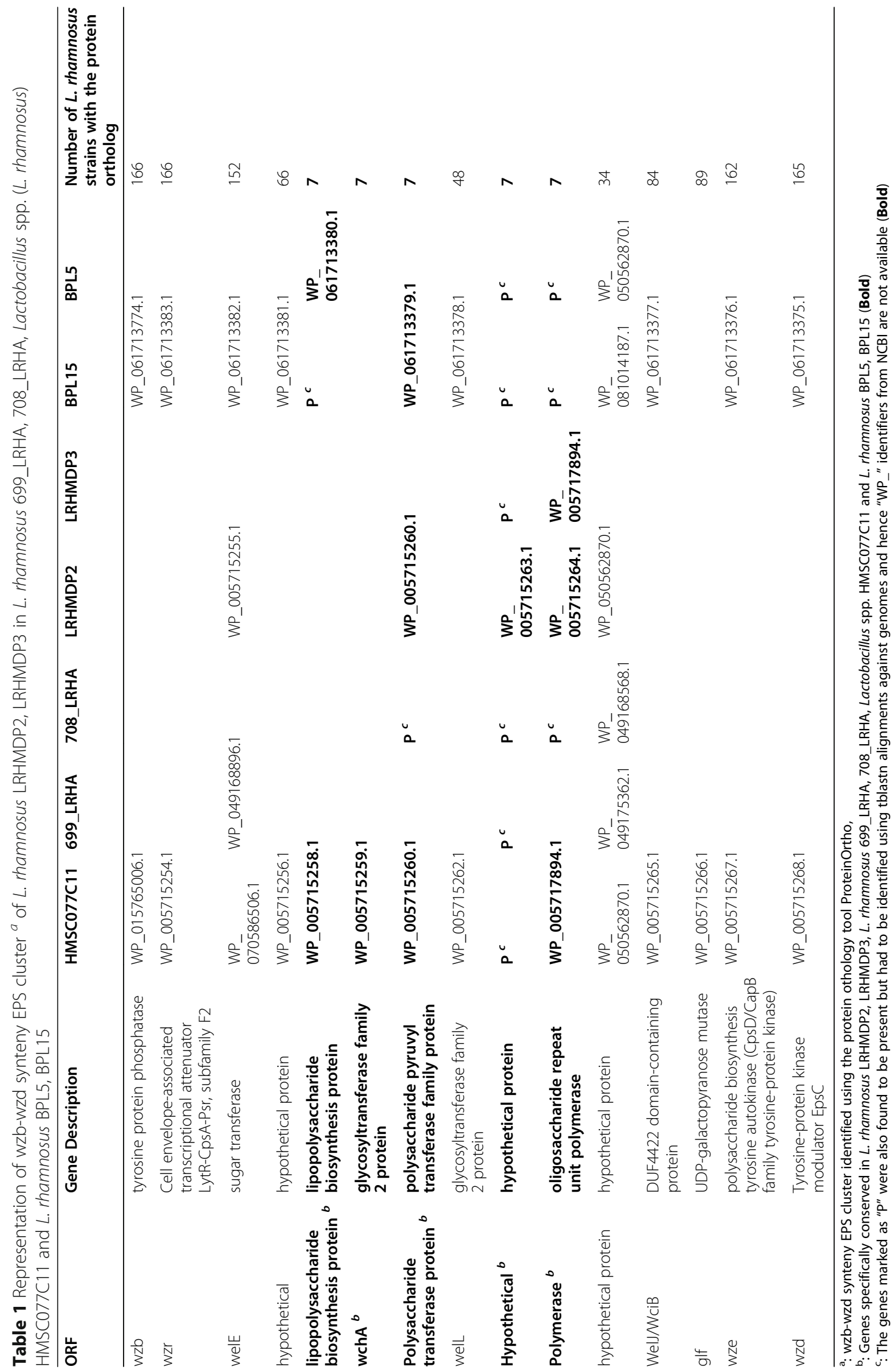




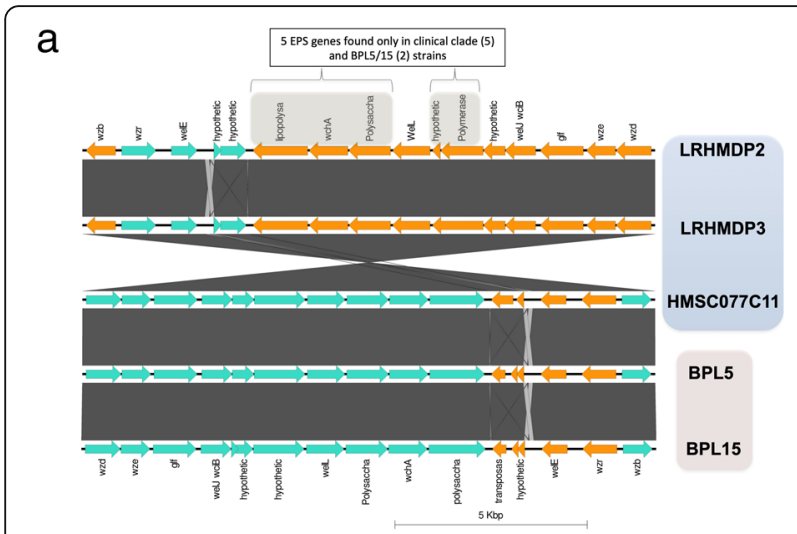

b

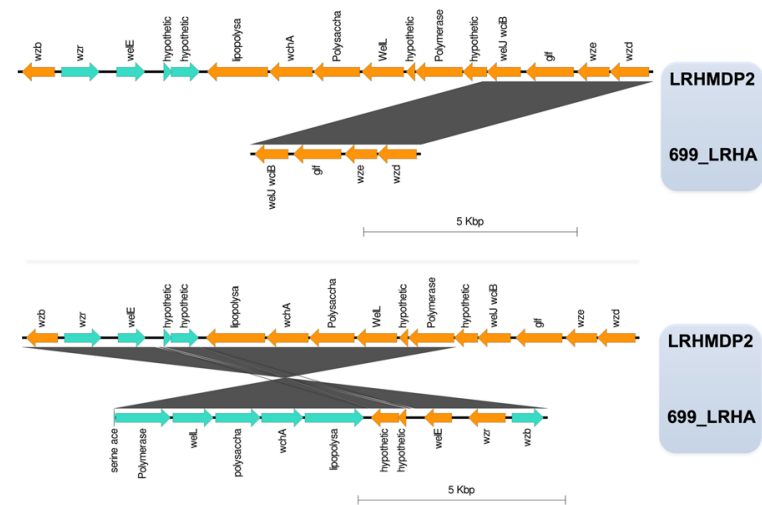

C

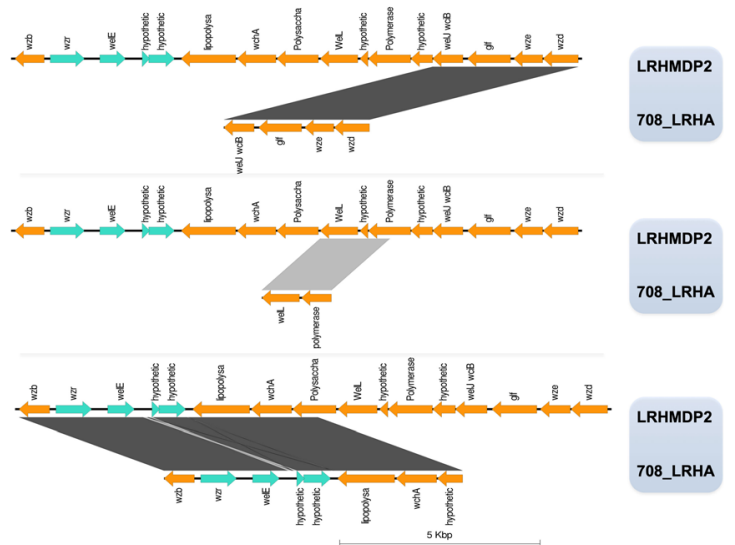

Fig. 2 Genomic organisation of wzb-wzd synteny EPS cluster of $L$. rhamnosus LRHMDP2, LRHMDP3, L. rhamnosus 699_LRHA, 708_LRHA, Lactobacillus spp. HMSC077C11 and L. rhamnosus BPL5/BPL15. Proteinortho, a standalone tool, was used to determine conservation of orthologous genes across selected $L$. rhamnosus isolates for a specific EPS gene cluster. Genomic organization of EPS cluster genes was ascertained using EasyFig, a python application for creating linear comparison figures of multiple genomic loci, within the 'wzbwzd' synteny EPS cluster locus. L. rhamnosus LRHMDP2, LRHMDP3, BPL5, BPL15 and Lactobacillus spp. HMSC077C11 on continuous contig (a), L. rhamnosus LRHMDP2, LRHMDP3 and 699_LRHA split on to 2 contigs (b). L. rhamnosus LRHMDP2, LRHMDP3 and 708_LRHA (split on to 3 contigs) (c)
Genomic features which distinguish the clinical clade of 5 L. rhamnosus isolates

Having established that the EPS cluster was common between the clinical clade of L. rhamnosus and strains BPL5 and BPL15, we sought to determine whether there were other features that were also common between these strains. Consistent with the substantial genomic distance between the clade of 5 clinical isolates and $L$. rhamnosus BPL5 and BPL15, no other gene-sets were specifically conserved between L. rhamnosus BPL5, BPL15 and the clade of 5, except for the near identical EPS cluster, with exclusivity defined by 5 EPS orthologs.

Using Proteinortho (protein sequences) and tblastn (genome sequence), 107 proteins were found to be orthologous across L. rhamnosus LRHMDP2, LRHM DP3, 699_LRHA, 708_LRHA and Lactobacillus spp. HMSC077C11 and to be absent in L. rhamnosus BPL5 and BPL15 (Table S2). Protein function could be attributed to 58 entities while 49 were categorised as hypothetical proteins (Table S2). These proteins separating the clade of 5 clinical isolates and L. rhamnosus BPL5 and BPL15 included an ABC-2 transporter permease, alpha/beta hydrolase, cytosine permease, DUF917 domain-containing protein, hydantoinase/oxoprolinase family protein, $\mathrm{N}$-acetyltransferase and SDR family NAD(P)-dependent oxidoreductase, all of which were also present in the majority of the other L. rhamnosus strains (Table 2). In addition, five genes of the six gene cassette of the Bacillus cereus phage defense system, BREX (bacteriophage exclusion), shown to confer resistance to integration of lysogenic (temperate) phages as well as replication of lytic phages $[26,27]$ could also be identified as a distinct feature of clade of 5 as compared to L. rhamnosus BPL5 and BPL15. BREX system orthologs of clade of 5 clinical isolates could be detected in other L. rhamnosus strains mainly from human origin and including vaginal isolates GR-1, 51B and DSM 14870 but not Lrh31 (Table S3). The clade of 5 BREX system orthologs could also be identified in most of the blood isolates (except for Lrh15 and Lrh47) including LRB1 and LRB-2 infant blood isolates and in the central venous catheter isolate from the $L$. rhamnosus GG clade. Similarly, presence of BREX system orthologs in clinical isolates from ICU patients could be grouped with other blood isolates. However, clade of 5 BREX system orthologs were not detected in the clinical isolates 526_LRHA, 541_LRHA, 769_LRHA, 879_LRHA, 943_LRHA and 988_LRHA from ICU patients, on a clade shared with blood isolate Lrh47. Absence of BREX system orthologs was also notable in isolates from infant saliva (L. rhamnosus 24) and from infant stools (L. rhamnosus L31, L34 and L35) (Table S3).

In L. rhamnosus isolates of human origin a link was evident between the presence or absence of clade of 5 
Table 2 Distinct functional protein orthologs associated with clade of 5 L. rhamnosus clinical isolates in comparison to L. rhamnosus BPL5, L. rhamnosus BPL15 and the rest of the L. rhmanouss genomes

\begin{tabular}{|c|c|c|}
\hline Description & Protein ID & \# L. rhamnosus Species \\
\hline 4-hydroxy-2-oxoglutarate aldolase / 2-dehydro-3-deoxyphosphogluconate aldolase & WP_005717709.1 & 77 \\
\hline$A B C-2$ transporter permease & WP_005715415.1 & 164 \\
\hline AbrB/MazE/SpoVT family DNA-binding domain-containing protein & WP_005715999.1 & 54 \\
\hline alpha-L-fucosidase & WP_005717725.1 & 81 \\
\hline alpha/beta hydrolase & WP_005715325.1 & 152 \\
\hline AraC family transcriptional regulator & WP_005714953.1 & 60 \\
\hline bacteriocin & WP_076638842.1 & 59 \\
\hline beta-galactosidase subunit alpha & WP_005714949.1 & 68 \\
\hline BREX system Lon protease-like protein BrxL & WP_005686196.1 & 91 \\
\hline BREX system P-loop protein BrxC & WP_005715305.1 & 89 \\
\hline BREX-1 system phosphatase PglZ type A & WP_005686195.1 & 87 \\
\hline carbohydrate PTS IIA component & WP_005715915.1 & 76 \\
\hline cytosine permease & WP_005715299.1 & 146 \\
\hline DNA helicase & WP_049168901.1 & 44 \\
\hline DUF1788 domain-containing protein (brxB) & WP_005684780.1 & 82 \\
\hline DUF1819 domain-containing protein (brxA) & WP_005684779.1 & 82 \\
\hline DUF2568 domain-containing protein & WP_005716418.1 & 53 \\
\hline DUF262 domain-containing protein sp. HMSC077C11 & WP_070586510.1 & 91 \\
\hline DUF2620 domain-containing protein & WP_005714931.1 & 37 \\
\hline DUF2992 domain-containing protein & WP_005685750.1 & 97 \\
\hline DUF917 domain-containing protein & WP_005715298.1 & 151 \\
\hline galactonate dehydratase & WP_005717713.1 & 77 \\
\hline GntR family transcriptional regulator & WP_005717726.1 & 78 \\
\hline helix-turn-helix domain-containing protein (Rgg/GadR/MutR family transcriptional regulator) & WP_032954331.1 & 45 \\
\hline HXXEE domain-containing protein & WP_005716405.1 & 80 \\
\hline hydantoinase/oxoprolinase family protein & WP_005715297.1 & 144 \\
\hline L-fucose isomerase & WP_005717692.1 & 74 \\
\hline membrane protein & WP_005714932.1 & 38 \\
\hline MerR family transcriptional regulator sp. HMSC077C11 & WP_070586464.1 & 53 \\
\hline metal-independent alpha-mannosidase & WP_005717706.1 & 73 \\
\hline N-acetyltransferase & WP_005715414.1 & 164 \\
\hline nucleoside-diphosphate sugar epimerase & WP_005717601.1 & 32 \\
\hline phosphotriesterase-related protein sp. HMSC077C11 & WP_070586459.1 & 41 \\
\hline PRD domain-containing protein & WP_032954335.1 & 21 \\
\hline PRD domain-containing protein & WP_005714930.1 & 37 \\
\hline pyridoxamine 5-phosphate oxidase family protein & WP_015764910.1 & 67 \\
\hline SDR family NAD(P)-dependent oxidoreductase & WP_005684771.1 & 152 \\
\hline SEC10/PgrA surface exclusion domain-containing protein sp. HMSC077C11 & WP_070586541.1 & 35 \\
\hline thymidylate synthase & WP_032954600.1 & 96 \\
\hline transaldolase & WP_005714910.1 & 37 \\
\hline transketolase & WP_005714895.1 & 43 \\
\hline type 1 glutamine amidotransferase domain-containing protein & WP_005717600.1 & 31 \\
\hline type III restriction protein res subunit (DEAD/DEAH box helicase) & WP_005716818.1 & 69 \\
\hline
\end{tabular}


Table 2 Distinct functional protein orthologs associated with clade of 5 L. rhamnosus clinical isolates in comparison to L. rhamnosus BPL5, L. rhamnosus BPL15 and the rest of the L. rhmanouss genomes (Continued)

\begin{tabular}{lll}
\hline Description & Protein ID & \# L. rhamnosus Species \\
\hline YhfX family PLP-dependent enzyme & WP_005714936.1 & 38 \\
\hline
\end{tabular}

BREX system orthologs and alpha-L-fucosidase, L-fucose isomerase and metal-independent alpha-mannosidase (Table 2, Table S3). The L. rhamnosus clinical isolates and other isolates of human origin with detectable BREX system orthologs also showed presence of alpha-Lfucosidase, L-fucose isomerase and metal-independent alpha-mannosidase. Exceptions included a clade shared between Lrh30, Lrh23, Lrh20 (blood isolates), 906 LRHA (clinical isolate) and GR-1 and 51B (vaginal isolates) which showed absence of alpha-L-fucosidase, Lfucose isomerase and metal-independent alphamannosidase despite detectable orthologs of clade of 5 BREX system orthologs. In contrast, another vaginal isolate L. rhamnosus DSM14870 showed presence of clade of 5 BREX system orthologs and alpha-L-fucosidase, Lfucose isomerase and metal-independent alphamannosidase. These genes were not detected in vaginal isolate Lrh31. This was similar to the profile for $L$. rhamnosus BPL5 and BPL15 and for clinical isolates from ICU patients (526_LRHA, 541_LRHA, 769_LRHA, 879_LRHA, 943_LRHA and 988_LRHA) and blood isolate Lrh 47. Alpha-L-fucosidase, L-fucose isomerase, metal-independent alpha-mannosidase and BREX system orthologs could be detected in other oral and salivary isolates, L. rhamnosus LRB and 313. In L. rhamnosus 24, an isolate from infant saliva, alpha-L-fucosidase and Lfucose isomerase were detected but BREX system orthologs were absent (Table 2, Table S3).

An identical pattern of distribution across the L. rhamnosus strains of human origin was also evident for HXXEE domain-containing protein and thymidylate synthase with alpha-L-fucosidase, L-fucose isomerase and metal-independent alpha-mannosidase and presence or absence of clade of 5 BREX system orthologs (Table 2, Table S3).

In contrast to the clade of $5, \mathrm{AbrB} / \mathrm{MazE} / \mathrm{SpoVT}$ family DNA-binding domain-containing protein from the toxin-antitoxin (TA) defence system was absent in the $L$. rhamnosus isolates from L. rhamnosus GG clade (including blood isolates, LR-CVC and oral isolate LRB), Lrh30, Lrh23 and Lrh20 (blood isolates), 906_LRHA (clinical isolate) and GR-1and 51B (vaginal isolate) and salivary isolate 313, but was present in infant salivary isolate $L$. rhamnosus 24. AbrB/MazE/SpoVT family DNA-binding domain-containing protein could not be detected in the clinical isolates from ICU patients, (526_LRHA, 541_ LRHA, 769_LRHA, 879_LRHA, 943_LRHA and 988_ LRHA) on a shared clade with blood isolate Lrh47.
However, the blood isolates, Lrh13, Lrh28, 186_LRHA, 214_LRHA, 390_LRHA on a clade shared with DSM 14870 (vaginal probiotic) and Lrh11, 319_LRHA, 784 LRHA and 893_LRHA showed presence of AbrB/MazE/ SpoVT family DNA-binding domain-containing protein from the clade of 5 clinical isolates.

A set of 14 potentially unique functional orthologs displayed orthologous conservation in the clade of 5 clinical isolates and were conserved across a limited number (between 1 and 7) of other L rhamnosus isolates. Notable being transcriptional regulators, RNA polymerase sigma-54 factor, XRE family transcriptional regulator, PRD domain-containing protein WP_005714928.1(earlier classified as $\mathrm{NtrC}$ transcriptional regulator [7]); also, Iron $\mathrm{ABC}$ transporter substrate-binding protein, iron $\mathrm{ABC}$ transporter permease, and two component sensor kinase with Ferric iron transporter, ImmA/IrrE family metallo-endopeptidase, IS91 family transposase, isochorismatase and ATP-binding protein (Table 3).

XRE family transcriptional regulator and ImmA/IrrE family metallo-endopeptidase were exclusively shared with L34, L35, L31 (infant stool isolates) and Lrh22 (blood isolate), RNA polymerase sigma-54 factor with L34, L35 and L31 and PRD domain-containing protein (NtrC transcription regulator) only with L34 and L35.

Iron $\mathrm{ABC}$ transporter substrate-binding protein, iron $\mathrm{ABC}$ transporter permease, two component sensor kinase with Ferric iron transporter and isochorismatase were highly conserved and exclusively shared orthologs between clade of 5 clinical isolates and L31 from infant faeces.

In addition to the clade of 5 clinical isolates, IS91 family transposase was detected only in JWHCO1 (L. rhamnosus strain 308 from saliva of a healthy female), AMC010 from stools of a healthy infant and E800 (from faeces). IS91 family transposase (identified earlier as a putative transposase) was found to be surrounded by a site-specific integrase, a phage portal protein and eight distinctive clade -specific hypothetical proteins (WP 005716827 - WP_005716850) and WP_032954616 (Table S2).

\section{Discussion}

We used the availability of a large number of L. rhamnosus genome sequences present in NCBI to uncover a unique, clinically relevant clade comprising $L$. rhamnosus LRHMDP2 and LRHMDP3 from dental pulp infection together with L. rhamnosus isolates 699_LRHA and 
Table 3 Exclusive set of unique functional protein orthologs in clade of $5 \mathrm{~L}$. rhamnosus clinical isolates in comparison to $\mathrm{L}$. rhamnosus BPL5, L. rhamnosus BPL15 and the rest of the L. rhamnosus genomes

\begin{tabular}{lll}
\hline Gene description & Protein IDs & \# L. rhamnosus Species \\
\hline ATP-binding protein & WP_005717598.1 & 9 \\
DUF4260 domain-containing protein & WP_080600050.1 & 8 \\
helix-turn-helix domain-containing protein (Rgg/GadR/MutR family transcriptional regulator) & WP_005717505.1 & 12 \\
ImmA/lrrE family metallo-endopeptidase & WP_005715904.1 & 9 \\
iron ABC transporter permease & WP_005716278.1 & 6 \\
iron ABC transporter substrate-binding protein & WP_005716273.1 & 6 \\
IS91 family transposase & WP_005717838.1 & 8 \\
isochorismatase & WP_005715911.1 & 6 \\
phage portal protein & WP_005716841.1 & 10 \\
PRD domain-containing protein & WP_005714928.1 & 7 \\
(NtrC transcription regulator) & & 8 \\
RNA polymerase sigma-54 factor & WP_005714927.1 \\
site-specific integrase & WP_080600030.1 \\
Two-component sensor kinase associated with ferric iron transporter & WP_005716270.1 \\
XRE family transcriptional regulator \\
(helix-turn-helix transcriptional regulator)
\end{tabular}

708_LRHA from bronchoalveolar lavage of a critical care patient [6]. The four L. rhamnosus clinical isolates shared closest genome neighbour gapped identity of 99.95\% and also shared the unique tyrosine protein phosphatase (wzb)-tyrosine-protein kinase modulator EpsC (wzd) synteny exopolysaccharide (EPS) cluster of L. rhamnosus LRHMDP2 and LRHMDP3 [7]. In addition, Lactobacillus spp. HMSC077C11, a clinical isolate from a neck abscess, was re-classified as Lactobacillus rhamnosus in the Genome Taxonomy Database (GTDB) [23]. This isolate occupied the same clade as $L$. rhamnosus LRHMDP2, LRHMDP3, 699_LRHA and 708_LRHA on the L. rhamnosus phylogenetic tree and shared the exclusive EPS cluster of $L$. rhamnosus LRHM DP2 and LRHMDP3.

A primary environmental niche for the clade of 5 has not been identified. Findings do not exclude an oral cavity source, particularly for isolates recovered from bronchoalveolar lavage but also from a neck abscess. A distinguishing genomic feature of this clade is an exopolysaccharide cluster. While a role for the exopolysaccharides of this clade in pathogenicity remains to be investigated, our preliminary studies, using partially purified polysaccharide extracted from isolate LRHM DP2, revealed perturbation of adaptive neurogenesis (data not shown). In context, there is a profound neural response in dental pulp tissue in response to microbial invasion of dentine [28]. Disruption of this adaptive response compromises defense allowing bacteria to invade dental pulp tissue. Modulation of neural function has been reported for bacterial polysaccharides [29] and this could represent an important aspect of the beneficial action of probiotic lactobacilli, particularly as there is limited evidence for effective colonisation of the adult human gut by these organisms [29].

Capsular polysaccharides and cell wall exopolysaccharides are significant for bacterial pathogenesis apart from potential contribution to the probiotic action of L. rhamnosus gut and vaginal isolates [18-22]. It is possible that differences in the relative orientation of EPS cluster genes within the clade of 5 could alter amounts and composition of exopolysaccharide in response to nutrient availability [30] and immune surveillance. The nature of the immune response and the property of adherence and biofilm formation has been shown to be influenced by minor variation in the structure of polysaccharide [31].

A finding in this study was the presence, in L. rhamnosus probiotic strains BPL5 and BPL15, of an inversion of the wzb-wzd EPS cluster present in L. rhamnosus LRHMDP2 and LRHMDP3. The inverted orientation of the EPS cluster and small differences in the functional orthologs could result in an altered exopolysaccharide in L. rhamnosus BPL5 and BPL15. These properties together with the added benefit of enhanced acidogenic capacity could be resultant contributors to the probiotic properties of L. rhamnosus BPL5 [32]. Divergence in the organisation of the EPS gene cluster and in composition of the exopolysaccharide among L. rhamnosus strains could hold significance for probiotic or pathological action $[1,5,18-22]$.

The differences between L. rhamnosus BPL5 and BPL15 and the clade of 5, that share near identical EPS clusters, were further investigated. In addition to the 
EPS cluster, niche adaptation has also been attributed to the accessory genome comprising pilus gene clusters, CRISPR - cas system genes, carbohydrate transport and metabolism genes, bacteriocin production and mobile genetic elements [1]. Comparative genome analysis between the clade of 5 and L. rhamnosus BPL 5 and BPL15 enabled identification of an exclusive set of 58 functionally identified protein orthologs. These included clade of 5 specific BREX system orthologs, alpha-L-fucosidase, Lfucose isomerase, metal-independent alpha-mannosidase, HXXEE domain-containing protein, thymidylate synthase and AbrB/MazE/SpoVT family DNA-binding domaincontaining protein.

The Bacillus cereus BREX system contributed one of the distinctive genomic features shared between the clade of 5 in comparison to L. rhamnosus BPL5 and BPL15. The BREX defense system in L. rhamnosus LRHMDP2 and LRHMDP3 consisted of a full length BREX-1 system adenine specific DNA-methytransferase PglX (1198aa) and a partial form of PglX (743aa) separated by a site-specific integrase. This system potentially confers resistance against a broad range of phages. The genomic arrangement is analogous to the BREX system type 1 of L. rhamnosus GG [27]. Five of the six-genecassette BREX defense system genes from the clade of 5 clinical isolates could be detected in many of the $L$. rhamnosus strains of human origin. These included multiple blood isolates, ICU isolates, probiotic strains and some of the oral and vaginal isolates. In the clade of 5, BREX system P-loop protein BrxC was identified as part of the exclusive set of functionally identified proteins. Although the P-loop-containing gene was conserved, shared homology in various BrxC from six BREX subtypes is low [27]. These findings imply importance for the BREX defense system across a spectrum of L. rhamnosus isolates. Another defense strategy, the CRISPR-cas system for control of phages, was shown to be absent in L. rhamnosus LRHMDP2 and LRHMDP3 [7]. Absence of the entire CRISPR-cas system was also apparent in $L$. rhamnosus Lc705, ATCC 8530, ATCC 7469 and 8 of 40 diverse L. rhamnosus isolates [1,33]. However, both of the phage resistance systems, CRISPR-cas and BREX, are present in L. rhamnosus GG $[27,33]$. The trade-off between presence and absence of CRISPR-cas for virulence is apparent in the human pathogen Streptococcus pneumoniae [34]. On the other hand, heterogeneity in PglX of the BREX system has been shown to control phase variation in bacterial defense systems to overcome toxic effects of certain genes [27].

Another distinctive feature of the clade of 5 clinical isolates is the antitoxin of the toxin-antitoxin (TA) defense system (AbrB/MazE/SpotVT family type DNA binding protein). This protein was absent from most other L. rhamnosus isolates. MazF and YaFQ TA systems have also been reported for L. rhamnosus isolates from clade 1 comprising isolates from blood, faeces and other clinical samples [1]. In E. coli, chromosomally located MazE antitoxin, a DNA binding protein, has been shown to wrap around the MazF toxin, an endonuclease [35]. Six additional TA systems were identified in L. rhamnosus intestinal, faecal and salivary isolates [36]. L. rhamnosus LRHMDP2 and LRHMDP3 were found to possesses five of the six TA systems whereas $L$. rhamnosus GG contained three of the six TA systems [36]. By reacting to multiple stress factors that a pathogen encounters in the host, TA systems are considered to modulate the host-pathogen interface [37]. Therefore, AbrB/MazE/SpotVT family type DNA binding proteins may signify a causal role for the clade of 5 in clinical pathology.

Another ortholog specific to the clade of 5 was alpha L-fucosidase, found to participate in the degradation of various fucosyl-glycoconjugates on epithelial cell surfaces and in blood group antigens, intestinal mucin and human milk [38]. Release of $\alpha$-linked fucose residues could provide a source of carbon for the clade of 5 in clinical conditions. The oral isolates $L$. rhamnosus LRHMDP2 and LRHMDP3 were identified as having an L-fucose fermenting phenotype with fuc clusters like those of L. rhamnosus GG and HN001 as opposed to the absence of fucose fermenting ability in the dairy isolates [39]. Alpha L-fucosidase along with metal-independent alpha-mannosidase could empower the clinical isolates to de-cap and harvest human glycans as evident in Streptococcus pneumoniae [40]. Also, within the clade of 5 , a GntR family transcriptional regulator was found adjacent to the gene encoding Alpha L-fucosidase. GntR family transcription regulators are termed as sugar transport system regulators in Streptococcus mutans [41]. These regulators were deduced to regulate multiple sugar transport genes, EPS production and biofilm formation [41]. Similarly, involvement of GntR type transcription factors in the regulation of the GalN/GalNAc utilization pathway is required for the virulence of Streptococcus suis serotype 2 [42]. Therefore, the clade of 5 - specific GntR family transcriptional regulators may play an important role in sugar transport systems, EPS and biofilm formation, in specified niches.

A set of 14 potentially unique functional orthologs could be closely associated with the clade of 5 clinical isolates. Notable inclusions are RNA polymerase sigma54 factor, XRE family transcriptional regulator, PRD domain-containing protein WP_005714928.1 (earlier classified as a NtrC transcription regulator [7]), iron $\mathrm{ABC}$ transporter substrate-binding protein, iron $\mathrm{ABC}$ transporter permease, a two component sensor kinase with ferric iron transporter, ImmA/IrrE family metalloendopeptidase, IS91 family transposase, isochorismatase 
and an ATP-binding protein. Comparative genomic analysis between $L$. rhamnosus LRHMDP2, LRHMDP3 and L. rhamnosus GG also identified RNA polymerase sigma-54 factor (RpoN), transcriptional regulators, NtrC and MutR, an iron $A B C$ transporter permease, iron $A B C$ transporter substrate and a two component sensor kinase with ferric iron transporter, as features of significance [7] within the clade of 5 .

The set of 14 potentially unique functional orthologs closely associated with the clade of 5 could not be found in most of the blood isolates (except for Lrh22) or in most of the clinical isolates (except for 944_LRHA) but were found in L. rhamnosus L34, L35 and L31 (isolates from infant faeces). XRE family transcriptional regulator and $\operatorname{ImmA} / \operatorname{IrrE}$ family metallo-endopeptidase were exclusively shared with L34, L35, L31 and Lrh22 (blood isolate) and RNA polymerase sigma-54 factor with L34, L35 and L31 and PRD domain-containing protein (NtrC transcriptional regulator) only with L34 and L35. L. rhamnosus L34, L35 (from a 40 day old infant) and L31 (from a 39 day old infant) were isolated from faeces of breast-fed infants from Thailand and were shown to have capacity to inhibit Clostridiodes difficile and exhibit antiinflammatory properties $[43,44]$. RNA polymerase sigma factor 54 (RpoN) was shown to regulate virulence genes, motility, quorum sensing and also tolerance to antibiotics in Psudomonas aeruginosa [45]. Similarly, transcription by sigma 54 holoenzyme was shown to be activated by phosphorylated NtrC oligomers [46]. The role of RNA polymerase sigma-54 factor and $\mathrm{NtrC}$ transcriptional regulator in the pathogenicity of the clade of $5 \mathrm{~L}$. rhamnosus clinical isolates is yet to be ascertained.

Potential significance could also be attached to the iron $\mathrm{ABC}$ transporter permease, iron $\mathrm{ABC}$ transporter substrate-binding protein, two component sensor kinase with ferric iron transporter and isochorismatase exclusively conserved between the clade of 5 and L. rhamnosus L31. In Acinetobacter baumannii a critical correlation has been shown between isochorismatase, siderophoremediated ferric iron acquisition and autophagy [47]. On the other hand, in Pseudomonas aeruginosa, isochorismatase is involved in the biosynthesis of an antimicrobial compound, phenazine, that may offer competitive advantage to this opportunistic pathogen [48].

IS91 family transposase, another unique functional ortholog closely associated with clade of 5 , was detected only in JWHC01 (L. rhamnosus strain 308 from saliva of a healthy female), AMC010 from stools of a healthy infant and E800 (from faeces). IS91 family transposase, uniquely employs rolling circle transposition in horizontal gene transfer $[49,50]$. IS91 insertion sequence has also been identified in Gram negative organisms and a link between IS91 family transposase and pathogenicity and virulence- related genes has been demonstrated for
E. coli $[51,52]$. In the clade of 5 , multiple genes designated as encoding hypothetical proteins are located adjacent to the gene encoding IS91 family transposase. Further studies will be required to elucidate the significance of this genic cluster.

\section{Conclusion}

In-silico analysis of the genomes of the clade of 5 clinical isolates highlighted a potentially unique complement of genes of clinical relevance. Except for the near identical EPS cluster, with exclusivity defined by five EPS orthologs, no other gene sets were specifically conserved between the clade of 5 and the probiotic strains, $L$. rhamnosus BPL5 and BPL15. Candidates emerging from the distinctive set of 58 genes identified in the clade of 5 include RNA polymerase sigma- 54 factor $(\mathrm{RpoN})$, transcriptional regulators, XRE family, NtrC, MutR, iron $\mathrm{ABC}$ transporter permease, iron $\mathrm{ABC}$ transporter substrate- binding protein and two component sensor kinase with Ferric iron transporter. Others include isochorismatase, DEAD/DEAH box helicase (Type III restriction protein res subunit) associated with remodelling and unwinding of RNA [53], helix-turn helix ( $\mathrm{HTH})$ domain containing proteins and PRD domain containing proteins as regulatory domains for PTS carbohydrate metabolism. The BREX defense system, toxin-antitoxin system and IS91 transposase and the surrounding group of phage-related and hypothetical proteins could signal the presence of novel defense systems [54] as components of pathogenicity islands in the clade of 5. Functional co-ordination between different defense systems in addition to the distinctive EPS cluster and associated alpha-L-fucosidase, L-fucose isomerase, metalindependent alpha-mannosidase, HXXEE domaincontaining protein and thymidylate synthase, may govern conditional opportunistic pathology associated with L. rhamnosus. Further studies could disclose whether the complex interplay between the 58 unique and potentially functional orthologs of the clade of 5 could serve as a model for opportunistic virulence .

\section{Methods}

\section{L. rhamnosus and Lactobacillus spp. genome sequences}

Genome sequences of L. rhamnosus LRHMDP2 and LRHMDP3 isolated from the early stages of dental pulp infection [7, 55], L. rhamnosus 699_LRHA and 708_ LRHA isolated from bronchoalveolar lavage [6], $L$. rhamnosus BPL5, originating from the vagina of a healthy women [24] and BPL15 and Lactobacillus spp. HMSC077C11 isolated from a neck abscess, formed part of this study (Table 4). All other L. rhamnosus genomes, which have been sequenced and deposited in NCBI, were used for a comprehensive comparative genomic analysis (Table S1). 


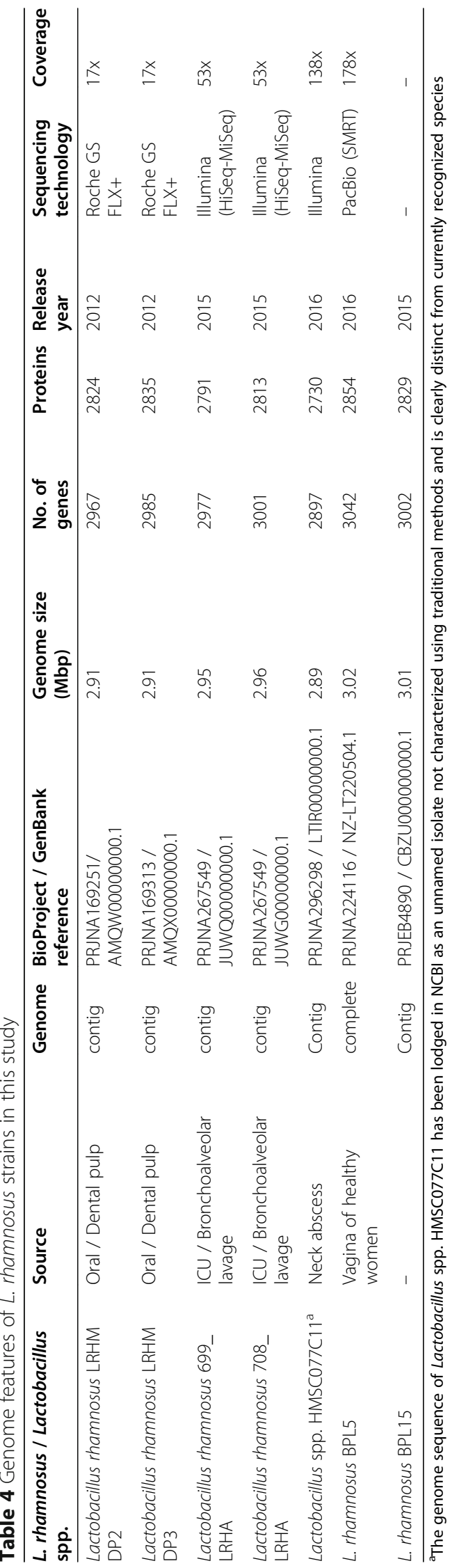




\section{L. rhamnosus phylogenetic tree}

We selected the GToTree tool to include Lactobacillus sp. HMSC077C11 along with the 4 clinical strains ( $L$. rhamnosus LRHMDP2, LRHMDP3, 699_LRHA and 708_LRHA) on the L. rhamnosus phylogeny tree. Lactobacillus sp. HMSC077C11 has been recently re-classified as L. rhamnosus HMSC077C11 in the Genome Taxonomy Database (GTDB) by genome-based species taxonomy study [23] and is not represented on the NCBI $L$. rhamnosus phylogeny tree. The phylogenetic tree built by the GToTree tool retains exact grouping as the NCBI L. rhamnosus phylogeny tree (https://www.ncbi.nlm.nih. gov/genome/?term=Lactobacillus+rhamnosus).

GToTree is the newly published bioinformatic tool, that can work with any custom hidden Markov Models (HMMs). It has also included 13 newly generated singlecopy gene (SCG) set HMMs for different lineages and levels of resolution, built based on searches of $\sim 12$, 000 high-quality bacterial and archaeal genomes. GToTree algorithm is more generic than its predecessors and the tool is highly scalable and user friendly. The tool accepts genome sequences and provides an alignment output and phylogenomic tree based on the specified hidden Markov Models (HMM) profiles [56]. From the available 15 single copy gene (SCG)-set HMMs in GToTree, we used the Firmicutes HMM SCG-set with 119 genes. The tree was viewed using the 'Interactive tree of life' web page (https://itol.embl.de/upload.cgi). The genbank, fasta and gff format files required for generating a phylogenetic tree and for subsequent comparative genomic analysis were downloaded using the NCBI web link https://www.ncbi.nlm.nih.gov/genome/doc/ftpfaq/

\#downloadservice (Table S1).

\section{Exopolysaccharide (EPS) cluster}

EPS cluster proteins from L. rhamnosus LRHMDP2 and LRHMDP3 were extracted from NCBI. Identical Protein groups (https://www.ncbi.nlm.nih.gov/ipg/) and the tool Proteinortho was used to identify conservation of orthologous genes across the other L. rhamnosus strains. Genomic organization of wzb-wzd synteny of the EPS cluster was ascertained using EasyFig, a python application for creating linear comparison figures of multiple genomic loci for displaying the similarities and differences within the 'wzb-wzd' synteny EPS cluster locus across the set of selected L. rhamnosus strains (LRHM DP2, LRHMDP3, 699_LRHA, 708_LRHA, BPL5 and BPL15 and Lactobacillus spp. HMSC077C11 [57]. The EasyFig tool provides a graphical user interface to upload individual Genbank formatted files. Pairwise blastn comparisons were conducted and the blast outputs were used to generate syntenical visualizations of the EPS clusters across selected L. rhamnosus strains together with gene orientations.

\section{Comparative genome-wide analysis}

Proteinortho tool was used to identify conservation of orthologous genes across selected L. rhamnosus isolates for both whole genome comparisons and for specific analysis of EPS cluster related genes [58]. Proteins specifically conserved in the clade of 5 clinical isolates were further confirmed for their specificity by tblastn alignments (protein sequences) against L. rhamnosus genomes, to identify false positives and negatives (due to missing gene annotations in individual genomes) and any other anomalies. Representation of the distinct set of protein orthologs based on the tool ProteinOrtho and tblastn differentiating clade of 5 from L. rhamnosus BPL5 and BPL15 was ascertained further in the remaining $L$. rhamnosus genome sequences.

\section{Supplementary information}

Supplementary information accompanies this paper at https://doi.org/10. 1186/s12864-020-07062-3.

Additional file 1: Table S1. List of L. rhamnosus strains downloaded for phylogenetic tree.

Additional file 2: Table S2. Protein orthologs exclusively shared between L. rhamnosus LRHMDP2, LRHMDP3, 699_LRHA, 708_LRHA and Lactobacillus spp. HMSC077C 11 in comparison to L. rhamnosus BPL5 and BPL15. Identified using ProteinOrtho and tblastn.

Additional file 3: Table S3.

\section{Abbreviations}

BREX: Bacteriophage exclusion; Cas: CRISPR associated system; CRIS PR: Clustered Regularly Interspaced Short Palindromic Repeat;

EPS: Exopolysaccharide; GRAS: Generally recognized as safe; HMM: Hidden Markov Models; ICU: Intensive care unit; SCG: Single copy gene; TA: Toxinantitoxin; Wzb: Tyrosine protein phosphatase; Wzd: Tyrosine-protein kinase modulator EpsC

\section{Acknowledgements}

NIL

\section{Authors' contributions}

Study Design (MN, ND, MW), data analysis (MN, ND), data interpretation (MN, $\mathrm{ND}, \mathrm{MW}$ ), drafting and revision of manuscript (MN, ND, MW, NH). The author(s) read and approved the final manuscript.

\section{Funding}

MW acknowledges funding from the Australian Government NCRIS program, the New South Wales State Government RAAP scheme and the University of New South Wales.

MN and NH acknowledge support from Westmead Centre for Oral Health.

\section{Availability of data and materials}

The genbank, fasta and gff format files required for generating a phylogenetic tree and for subsequent comparative genomics analysis were downloaded using the NCBI web link https://www.ncbi.nlm.nih.gov/ genome/doc/ftpfaq/\#downloadservice (Table S1).

Further data analysis is supported by supplementary files.

Ethics approval and consent to participate

Not Applicable.

Consent for publication

All authors have approved the manuscript and agree with its publication on BMC Genomics. 


\section{Competing interests}

The authors declare that the research was conducted in the absence of any commercial or financial relationships that could be construed as a potential conflict of interest.

\section{Author details}

'Institute of Dental Research, Westmead Centre for Oral Health, Westmead Hospital, Sydney, New South Wales, Australia. ${ }^{2}$ Westmead Institute for Medical Research, The University of Sydney, Sydney, New South Wales, Australia. ${ }^{3}$ The School of Dentistry, the Faculty of Medicine and Health, The University of Sydney, Sydney, New South Wales, Australia. ${ }^{4}$ Systems Biology Initiative, School of Biotechnology and Biomolecular Sciences, The University of New South Wales, Sydney, New South Wales, Australia. ${ }^{5}$ School of Biotechnology and Biomolecular Sciences, The University of New South Wales, Sydney, New South Wales, Australia. ${ }^{6}$ Ramaciotti Centre for Genomics, The University of New South Wales, Sydney, New South Wales, Australia.

Received: 4 May 2020 Accepted: 10 September 2020

Published online: 24 September 2020

\section{References}

1. Ceapa C, Davids M, Ritari J, Lambert J, Wels M, Douillard FP, Smokvina T, de Vos WM, Knol J, Kleerebezem M. The variable regions of Lactobacillus rhamnosus genomes reveal the dynamic evolution of metabolic and hostadaptation repertoires. Genome Biol Evol. 2016;8(6):1889-905.

2. Douillard FP, Ribbera A, Kant R, Pietila TE, Jarvinen HM, Messing M, Randazzo CL, Paulin L, Laine P, Ritari J, et al. Comparative genomic and functional analysis of 100 Lactobacillus rhamnosus strains and their comparison with strain GG. PLoS Genet. 2013;9(8):e1003683.

3. Kant R, Rintahaka J, Yu X, Sigvart-Mattila P, Paulin L, Mecklin JP, Saarela M, Palva A, von Ossowski I. A comparative pan-genome perspective of nicheadaptable cell-surface protein phenotypes in Lactobacillus rhamnosus. PLoS One. 2014;9(7):e102762.

4. Rossi F, Amadoro C, Colavita G. Members of the Lactobacillus Genus Complex (LGC) as Opportunistic Pathogens: A Review. Microorganisms. 2019;7(5).

5. Nissila E, Douillard FP, Ritari J, Paulin L, Jarvinen HM, Rasinkangas $P$, Haapasalo K, Meri S, Jarva H, de Vos WM. Genotypic and phenotypic diversity of Lactobacillus rhamnosus clinical isolates, their comparison with strain GG and their recognition by complement system. PLoS One. 2017; 12(5):e0176739.

6. Roach DJ, Burton JN, Lee C, Stackhouse B, Butler-Wu SM, Cookson BT, Shendure J, Salipante SJ. A year of infection in the intensive care unit: prospective whole genome sequencing of bacterial clinical isolates reveals cryptic transmissions and novel microbiota. PLoS Genet. 2015;11(7): e1005413.

7. Nadkarni MA, Chen Z, Wilkins MR, Hunter N. Comparative genome analysis of Lactobacillus rhamnosus clinical isolates from initial stages of dental pulp infection: identification of a new exopolysaccharide cluster. PLoS One. 2014; 9(3):e90643.

8. Nadkarni MA, Simonian MR, Harty DW, Zoellner H, Jacques NA, Hunter N. Lactobacilli are prominent in the initial stages of polymicrobial infection of dental pulp. J Clin Microbiol. 2010:48(5):1732-40.

9. Kianoush N, Adler CJ, Nguyen KA, Browne GV, Simonian M, Hunter N. Bacterial profile of dentine caries and the impact of $\mathrm{pH}$ on bacterial population diversity. PLoS One. 2014;9(3):e92940.

10. Byun $\mathrm{R}$, Nadkarni MA, Chhour KL, Martin FE, Jacques NA, Hunter N Quantitative analysis of diverse Lactobacillus species present in advanced dental caries. J Clin Microbiol. 2004:42(7):3128-36.

11. Chhour KL, Nadkarni MA, Byun R, Martin FE, Jacques NA, Hunter N. Molecular analysis of microbial diversity in advanced caries. J Clin Microbiol. 2005;43(2):843-9.

12. Gross EL, Leys EJ, Gasparovich SR, Firestone ND, Schwartzbaum JA, Janies DA, Asnani K, Griffen AL. Bacterial 165 sequence analysis of severe caries in young permanent teeth. J Clin Microbiol. 2010;48(11):4121-8

13. Martin FE, Nadkarni MA, Jacques NA, Hunter N. Quantitative microbiological study of human carious dentine by culture and real-time PCR: association of anaerobes with histopathological changes in chronic pulpitis. J Clin Microbiol. 2002;40(5):1698-704.

14. Biswas S, Biswas I. Complete Genome Sequence of Lactobacillus rhamnosus Strain LRB. Genome Announcements. 2016;4(6).
15. Cannon JP, Lee TA, Bolanos JT, Danziger LH. Pathogenic relevance of Lactobacillus: a retrospective review of over 200 cases. Eur J Clin Microbiol Infect Dis. 2005;24(1):31-40.

16. Harty DW, Oakey HJ, Patrikakis M, Hume EB, Knox KW. Pathogenic potential of lactobacilli. Int J Food Microbiol. 1994;24(1-2):179-89.

17. Saxelin M, Tynkkynen S, Mattila-Sandholm T, de Vos WM. Probiotic and other functional microbes: from markets to mechanisms. Curr Opin Biotechnol. 2005;16(2):204-11.

18. Lebeer S, Verhoeven TL, Francius G, Schoofs G, Lambrichts I, Dufrene Y, Vanderleyden J, De Keersmaecker SC. Identification of a gene cluster for the biosynthesis of a long, Galactose-rich exopolysaccharide in Lactobacillus rhamnosus GG and functional analysis of the priming Glycosyltransferase. Appl Environ Microbiol. 2009;75(11):3554-63.

19. Gorska S, Schwarzer M, Jachymek W, Srutkova D, Brzozowska E, Kozakova H, Gamian A. Distinct immunomodulation of bone marrow-derived dendritic cell responses to Lactobacillus plantarum WCFS1 by two different polysaccharides isolated from Lactobacillus rhamnosus LOCK 0900. Appl Environ Microbiol. 2014;80(20):6506-16.

20. Marcotte $H$, Krogh Andersen $K$, Lin Y, Zuo F, Zeng Z, Larsson PG, Brandsborg E, Bronstad G, Hammarstrom L. Characterization and complete genome sequences of L. rhamnosus DSM 14870 and L. gasseri DSM 14869 contained in the EcoVag((R)) probiotic vaginal capsules. Microbiol Res. 2017; 205:88-98.

21. Petrova MI, Macklaim JM, Wuyts S, Verhoeven T, Vanderleyden J, Gloor GB, Lebeer S, Reid G. Comparative genomic and phenotypic analysis of the vaginal probiotic Lactobacillus rhamnosus GR-1. Front Microbiol. 2018;9: 1278 .

22. Peant B, LaPointe G, Gilbert C, Atlan D, Ward P, Roy D. Comparative analysis of the exopolysaccharide biosynthesis gene clusters from four strains of Lactobacillus rhamnosus. Microbiology. 2005;151(Pt 6):1839-51.

23. Wittouck S, Wuyts S, Meehan CJ, van Noort V, Lebeer S. A GenomeBased Species Taxonomy of the Lactobacillus Genus Complex. mSystems. 2019;4(5).

24. Chenoll E, Codoner FM, Martinez-Blanch JF, Ramon D, Genoves S, Menabrito M. Complete Genome Sequence of Lactobacillus rhamnosus Strain BPL5 (CECT 8800), a Probiotic for Treatment of Bacterial Vaginosis. Genome Announcements. 2016;4(2)

25. Bambace MF, Alvarez MV, Moreira MDR. Novel functional blueberries: Fructo-oligosaccharides and probiotic lactobacilli incorporated into alginate edible coatings. Food Res Int. 2019;122:653-60.

26. Barrangou R, van der Oost J. Bacteriophage exclusion, a new defense system. EMBO J. 2015:34(2):134-5.

27. Goldfarb T, Sberro H, Weinstock E, Cohen O, Doron S, Charpak-Amikam Y, Afik S, Ofir G, Sorek R. BREX is a novel phage resistance system widespread in microbial genomes. EMBO J. 2015;34(2):169-83.

28. Farahani RM, Rezaei-Lotfi S, Simonian M, Xaymardan M, Hunter N. Neural microvascular pericytes contribute to human adult neurogenesis. J Comp Neurol. 2019;527(4):780-96

29. Hyland NP, Cryan JF. Microbe-host interactions: influence of the gut microbiota on the enteric nervous system. Dev Biol. 2016;417(2):182-7.

30. Wicken AJ, Ayres A, Campbell LK, Knox KW. Effect of growth conditions on production of rhamnose-containing cell wall and capsular polysaccharides by strains of Lactobacillus casei subsp. rhamnosus. J Bacteriol. 1983;153(1):84-92.

31. Lebeer S, Claes I, Tytgat HL, Verhoeven TL, Marien E, von Ossowski I, Reunanen J, Palva A, Vos WM, Keersmaecker SC, et al. Functional analysis of Lactobacillus rhamnosus GG pili in relation to adhesion and immunomodulatory interactions with intestinal epithelial cells. Appl Environ Microbiol. 2012;78(1):185-93.

32. Chenoll E, Moreno I, Sanchez M, Garcia-Grau I, Silva A, Gonzalez-Monfort M, Genoves S, Vilella F, Seco-Durban C, Simon C, et al. Selection of new probiotics for endometrial health. Front Cell Infect Microbiol. 2019;9:114.

33. Kankainen M, Paulin L, Tynkkynen S, von Ossowski I, Reunanen J, Partanen $P$, Satokari R, Vesterlund S, Hendrickx AP, Lebeer $S$, et al. Comparative genomic analysis of Lactobacillus rhamnosus GG reveals pili containing a human- mucus binding protein. Proc Natl Acad Sci U S A. 2009;106(40): 17193-8.

34. Bikard D, Hatoum-Aslan A, Mucida D, Marraffini LA. CRISPR interference can prevent natural transformation and virulence acquisition during in vivo bacterial infection. Cell Host Microbe. 2012;12(2):177-86.

35. Chan WT, Espinosa M, Yeo CC. Keeping the wolves at bay: antitoxins of prokaryotic type II toxin-antitoxin systems. Front Mol Biosci. 2016;3:9. 
36. Klimina KM, Kjasova DK, Poluektova EU, Krugel H, Leuschner Y, Saluz HP, Danilenko VN. Identification and characterization of toxin-antitoxin systems in strains of Lactobacillus rhamnosus isolated from humans. Anaerobe. 2013;22:82-9.

37. Lobato-Marquez D, Diaz-Orejas R, Garcia-Del Portillo F. Toxin-antitoxins and bacterial virulence. FEMS Microbiol Rev. 2016:40(5):592-609.

38. Ashida H, Miyake A, Kiyohara M, Wada J, Yoshida E, Kumagai H, Katayama T, Yamamoto K. Two distinct alpha-L-fucosidases from Bifidobacterium bifidum are essential for the utilization of fucosylated milk oligosaccharides and glycoconjugates. Glycobiology. 2009;19(9):1010-7.

39. Becerra JE, Yebra MJ, Monedero V. An L-Fucose operon in the probiotic Lactobacillus rhamnosus GG is involved in adaptation to gastrointestinal conditions. Appl Environ Microbiol. 2015;81(11):3880-8.

40. Hobbs JK, Pluvinage B, Robb M, Smith SP, Boraston AB. Two complementary alpha-fucosidases from Streptococcus pneumoniae promote complete degradation of host-derived carbohydrate antigens. J Biol Chem. 2019.

41. Li Z, Xiang Z, Zeng J, Li Y, Li J. A GntR family transcription factor in Streptococcus mutans regulates biofilm formation and expression of multiple sugar transporter genes. Front Microbiol. 2018;9:3224.

42. Zhang H, Ravcheev DA, Hu D, Zhang F, Gong X, Hao L, Cao M, Rodionov DA, Wang C, Feng Y. Two novel regulators of $\mathrm{N}$-acetyl-galactosamine utilization pathway and distinct roles in bacterial infections. MicrobiologyOpen. 2015;4(6):983-1000.

43. Boonma P, Spinler JK, Qin X, Jittaprasatsin C, Muzny DM, Doddapaneni H, Gibbs R, Petrosino J, Tumwasorn S, Versalovic J. Draft genome sequences and description of Lactobacillus rhamnosus strains L31, L34, and L35. Stand Genomic Sci. 2014;9(3):744-54.

44. Boonma P, Spinler JK, Venable SF, Versalovic J, Tumwasorn S. Lactobacillus rhamnosus L34 and Lactobacillus casei L39 suppress Clostridium difficileinduced IL-8 production by colonic epithelial cells. BMC Microbiol. 2014;14: 177.

45. Lloyd MG, Lundgren BR, Hall CW, Gagnon LB, Mah TF, Moffat JF, Nomura $\mathrm{CT}$. Targeting the alternative sigma factor RpoN to combat virulence in Pseudomonas aeruginosa. Sci Rep. 2017;7(1):12615.

46. Yan D, Kustu S. "Switch I" mutant forms of the bacterial enhancer-binding protein NtrC that perturb the response to DNA. Proc Natl Acad Sci U S A. 1999;96(23):13142-6

47. Wang Y, Zhang K, Shi X, Wang C, Wang F, Fan J, Shen F, Xu J, Bao W, Liu M, et al. Critical role of bacterial isochorismatase in the autophagic process induced by Acinetobacter baumannii in mammalian cells. FASEB J. 2016; 30(10):3563-77.

48. Parsons JF, Calabrese K, Eisenstein E, Ladner JE. Structure and mechanism of Pseudomonas aeruginosa PhzD, an isochorismatase from the phenazine biosynthetic pathway. Biochemistry. 2003;42(19):5684-93.

49. del Pilar G-BM, Bernales I, Mendiola MV, de la Cruz F. Single-stranded DNA intermediates in IS91 rolling-circle transposition. Mol Microbiol. 2001;39(2): 494-501.

50. Wawrzyniak P, Plucienniczak G, Bartosik D. The different faces of rollingcircle replication and its multifunctional initiator proteins. Front Microbiol. 2017:8:2353

51. Garcillan-Barcia MP, de la Cruz F. Distribution of IS91 family insertion sequences in bacterial genomes: evolutionary implications. FEMS Microbiol Ecol. 2002:42(2):303-13.

52. Mahillon J, Chandler M. Insertion sequences. Microbiol Mol Biol Rev. 1998; 62(3):725-74.

53. Gilman B, Tijerina P, Russell R. Distinct RNA-unwinding mechanisms of DEAD-box and DEAH-box RNA helicase proteins in remodeling structured RNAs and RNPs. Biochem Soc Trans. 2017:45(6):1313-21.

54. Koonin EV, Makarova KS, Wolf YI. Evolutionary genomics of defense Systems in Archaea and Bacteria. Annu Rev Microbiol. 2017;71:233-61.

55. Chen Z, Wilkins MR, Hunter N, Nadkarni MA. Draft Genome Sequences of Two Clinical Isolates of Lactobacillus rhamnosus from Initial Stages of Dental Pulp Infection. Genome Announcements. 2013;1(1).

56. Lee MD. GToTree: a user-friendly workflow for phylogenomics. Bioinformatics. 2019

57. Sullivan MJ, Petty NK, Beatson SA. Easyfig: a genome comparison visualizer. Bioinformatics. 2011;27(7):1009-10.

58. Lechner M, Findeiss S, Steiner L, Marz M, Stadler PF, Prohaska SJ. Proteinortho: detection of (co-)orthologs in large-scale analysis. BMC Bioinformatics. 2011:12:124.

\section{Publisher's Note}

Springer Nature remains neutral with regard to jurisdictional claims in published maps and institutional affiliations.
Ready to submit your research? Choose BMC and benefit from:

- fast, convenient online submission

- thorough peer review by experienced researchers in your field

- rapid publication on acceptance

- support for research data, including large and complex data types

- gold Open Access which fosters wider collaboration and increased citations

- maximum visibility for your research: over $100 \mathrm{M}$ website views per year

At BMC, research is always in progress.

Learn more biomedcentral.com/submissions 